\title{
Design of Tire Surface Check System with the Laser Sensor
}

\author{
Sung-Hyun Baek* and Jong-Wook Jang* \\ *Department of Computer Engineering, Dong-Eui University \\ Busan, Korea \\ Smartsma@naver.com
}

\begin{abstract}
Vehicle tire should support the vehicle weight, deliver its own driving force and the braking force to the road surface, alleviate the impact applied from the vehicle, and switch hemistich progression such as the very important role in the automobile parts. The tire is one of the important parts of the car; a small error can affect the driver and passenger's safety. There are a number of problems with the tires, but generally we can divide them into two kinds: normal wear and abnormal wear. The normal tire wear occurs by driving, it can be replaced when mileage reaches recommended distance. The abnormal tire wear, unlike the usual wear, wears down tires unevenly or wears out only part of the tires. Until now, the method to check abnormal tire wear is that a mechanic checks tires with naked eyes and hands to confirm uneven tire wear. However, drivers are embarrassed when facing varied and vague rules of uneven wear, even for each tire. In this paper, the researchers have designed a system to check each tire wear by using one or two laser sensors in order to make up for this weakness and overcome the limits of subjective judgment and obtain objective, accurate judgment.
\end{abstract}

Keywords: vehicle tire, abnormal tire wear, laser sensor, android, normal tire wear, tire

\section{Introduction}

Vehicle tires utilize the rubber elasticity and air contractibility to support car weight, deliver driving force and braking power of a car to the road surface, as well as relieve the impact from road surface in order to switch or maintain the traveling direction of a car. The tire plays a very important role in driving.

The life span of this important tire depends on the degree of vehicle tire wear. Wear life of vehicle tire is directly related to vehicle maintenance cost. Drivers usually have great complaints when tires are replaced often. Also, when tire wear is advanced, there will be a crack or damage on the tire. If the car in this state continues to drive, it has longer braking distance and slips more often, which increases the risk of accidents. Seriously worn-out tires cause "hydroplaning", and when it happens, the car slips easily. Depending on the wear degree of tire, the probability of car accidents increases and it leads to serious accidents, so checking tire wear is essential. Tire wear is a very important element for driving and car accidents.

In this paper, the researchers aim to overcome these shortcomings. A system is designed to check wear degree and any abnormal condition of each tire by using multiple laser sensors so that drivers satisfy their right to be informed from objective and correct measurement of the sensors and machine. 


\section{System Design}

The tire surface check system in the paper is largely divided into two parts. One is the scanner to measure the tire wear, and the other is the integrated control server to measure the values, inform the driver, and save the data [Figure 1].

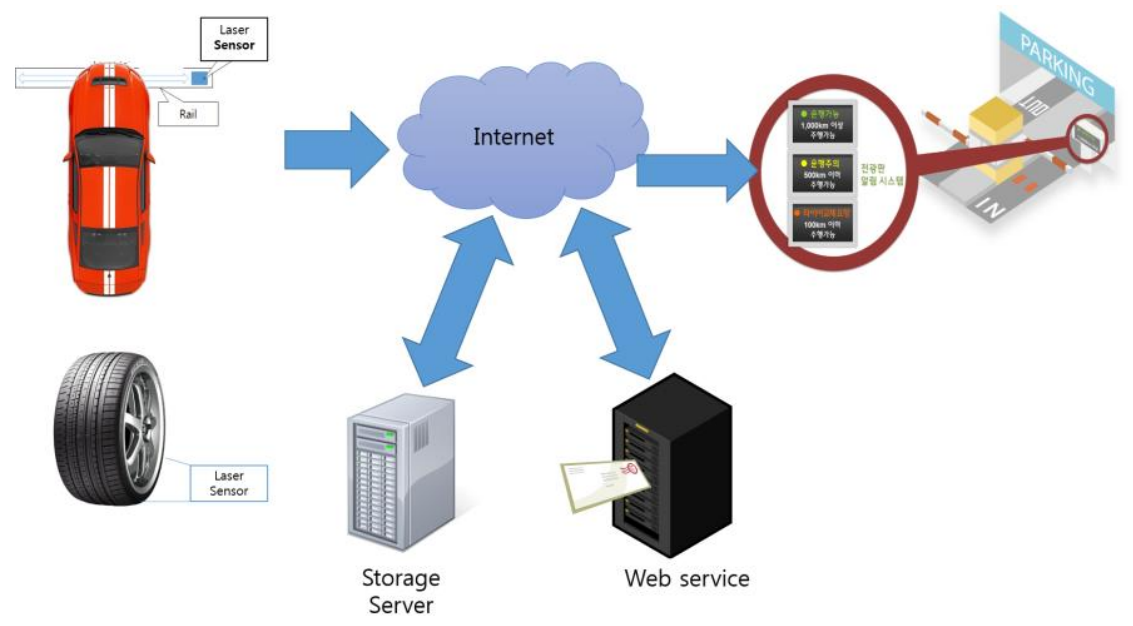

Figure 1. System Design

\subsection{The Scanner Design}

The scanner collects the tire information from several laser sensors attached on the road and tire number data side tire. When a car passes on top of the sensor region, these laser sensors check the tire surface and send the data to the main MCU using I/O channel. The laser sensor and the main MCU are physically connected to operate. The configuration of the scanner consists of three parts; the laser sensors, the part to handle the data from the sensors, and the part to send the data to the integrated control server. Because cars basically have four tires, and because each tire has a different tire hole from every tire maker, we will check the domain of at least 4 places. We equip one laser sensor in each area and will check the load condition of tire [Figure 2]. Next, we are using a laser range sensor to know the information of the tire and check the tire rolling wheels by installing a laser range sensor to the tire by side [Figure 3].

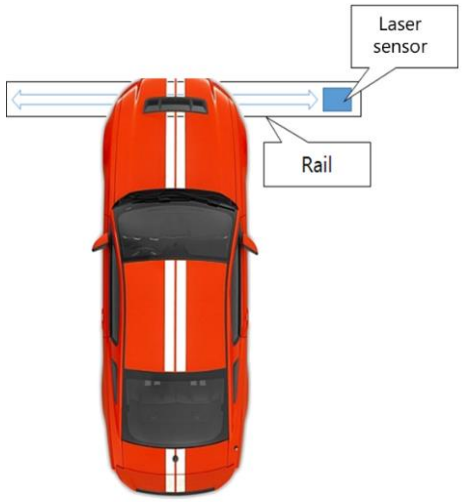

Figure 2. Laser Sensor Part of Vehicle

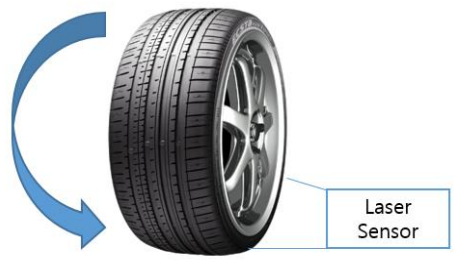

Figure 3. Laser Sensor Part of Tire 


\subsection{The Scanner Design}

LCD should be huge so drivers can see the tire information in real time when they enter a gas station or a pier. The large LCD shows signs like normal, warning, and danger to help drivers easily know their tire status. In addition, the warning screen informs drivers how many kilometers are left before they need to change the tire so that they can be informed of the time to change the tire [Figure 4-5].

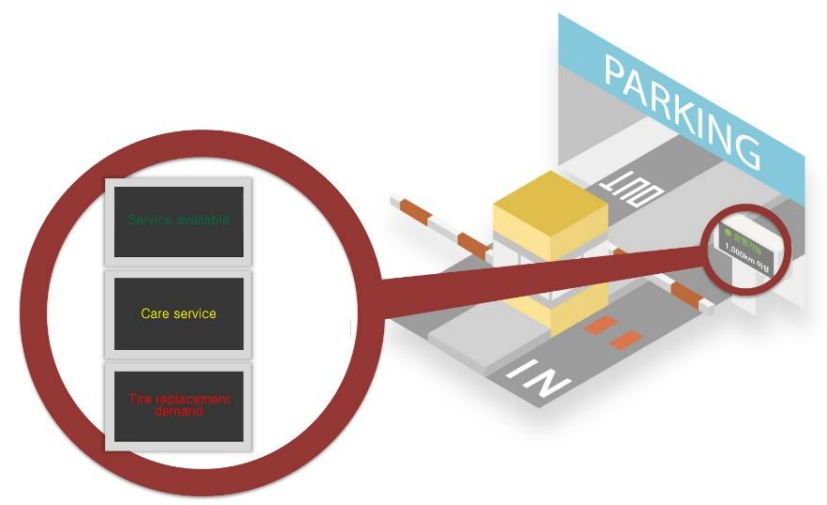

Figure 4. Display the LCD

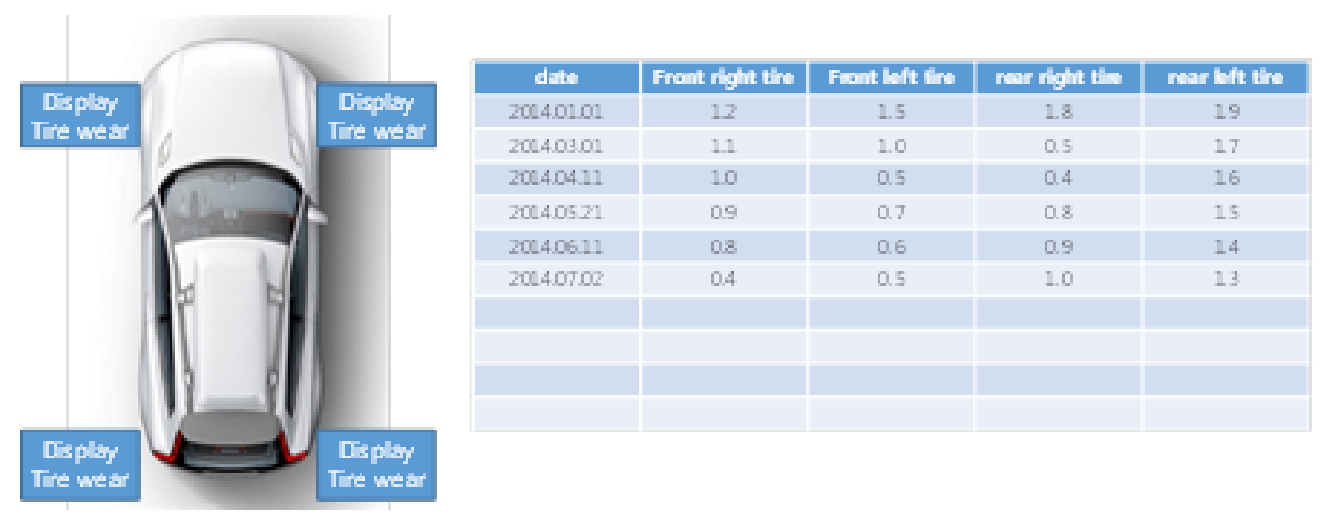

Figure 5. Display PC Screen

\subsection{Integrated Control Server Design}

The integrated control server saves the tire information received from uneven wear scanners and provides them to the users through their smart phone application.

The integrated control server has two servers to provide its service: uneven wear check server and web service server [Figure 6]. The integrated control server has fore process [Figure 7].

\begin{tabular}{|c|}
\hline OS : Linux \\
\hline DBMS : Oracle \\
\hline Framework: Spring \\
\hline UI Report : JSP, Jquery, MyBuilder \\
\hline
\end{tabular}

Figure 6. Integrated Control Server Design 


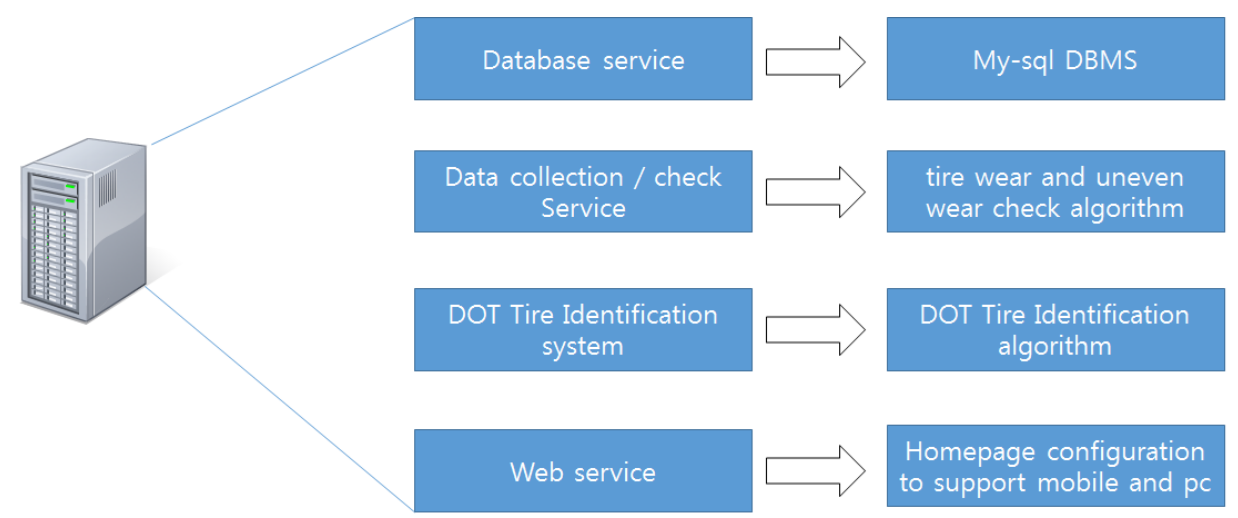

Figure 7. Process of Server

\section{Conclusion}

In this paper, the researchers have designed a tire surface check system with laser sensors in order to check the normal wear and abnormal wear of tires.

Unlike the existing methods, this system uses laser sensors to determine current abnormal wear state objectively and also check the degree of tire wear simultaneously. Thus, the users can be provided with more accurate service than before. Furthermore, the drivers can check their tire wear whenever and wherever because of the smart phone application which provides the tire data. However, to implement a system like this, there will also be problems in receiving and handling all the data related to all the tires of vehicles. Also, the wear degree should be confirmed by the depth of the tire tread groove, but we don't have a measure to gauge the tread depth with the laser sensors.

The future subject will be the application and establishment of the system. It will apply and realize the system designed in this paper to build a perfect system through real tests and debugging. Furthermore, it will establish a better system by researching the data related to every tire of all vehicles. By connecting to OBD-II, the automotive network, and real-time alarm for tire replacement time, we can build the system to secure maximum safety for tire wear.

\section{Acknowledgements}

This research was supported by the Brain Busan 21 Project in 2014 and Nurimaru R\&BD Project (Busan IT Industry Promotion Agency) in 2014

\section{References}

[1] S. S. Chung, W. W. Jung, S. J. Lee, B. J. Koh and Y. S. Choi, "Evaluation and Analysis of Wheel alignment effecting on Tire Uneven Wear", The Korean Society of Mechanical Engineers 2007 spring conference, vol. 5, (2007), pp. 318-322.

[2] S.-J. Park, "Car tire care, do your car is safe ?", "http://blog.skenergy.com/738"

[3] Y.-g. Song, "abnormal wear of the tire and the cause, (1991), pp. 46-48.

[4] H. Nowakowska, M. Jasinski, P. S. Debicki and J. Mizeraczyk, "Numerical analysis and optimization of power coupling efficiency in waveguide-based microwave plasma source", IEEE Transactions on Plasma Science [Online], vol. 39, no. 10, pp. 1935-1942, 2011, Available: http://ieeexplore.ieee.org/ $\mathrm{xpl} /$ articleDetails.jsp?arnumber $=6003795$ tire DOT,

[5] http://kr.goodyear.com/labs/education_reading.asp

[6] J. H. Kim and Jae C. Sim, "DOT Classification Code Extraction through 3 Dimensional Features", Proceedings of the 28th KISS Fall Conference, (2011), pp. 394-396. 
[7] K. S. Lee and J. T. Won, "A Identification of Tire Moldnumber using 3 Dimension Data", Institute of Electrical Engineers, vol. 54, no.10, (2005), pp. 595-603.

[8] Y. O. Kim, "tire Automotive Safety Management Trends", The Monthly Technology and Standard, (2009), pp. 40-44.

[9] J. Byun, Y. S. Cho and S. H. Kim, "Road Recognition based Extended Kalman Filter with Multi-Camera and LRF”, Korea Robotics Society, vol. 6, no. 2, (2011), pp.182-188. 
International Journal of Control and Automation Vol.8, No.3 (2015) 